\title{
OJAPr Editorial
}

\section{Miroslav Joler}

Faculty of Engineering, University of Rijeka, Rijeka, Croatia. Email: mjoler@riteh.hr

Received May 20 ${ }^{\text {th }}$, 2013; Revised June 21 ${ }^{\text {st }}$, 2013; Accepted June $27^{\text {th }}, 2013$

Copyright (C) 2013 Miroslav Joler. This is an open access article distributed under the Creative Commons Attribution License, which permits unrestricted use, distribution, and reproduction in any medium, provided the original work is properly cited.

On behalf of Scientific Research Publishing (SCIRP) Open Journal of Antennas and Propagation (OJAPr) editorial board and staff, it is my honor to write this editorial in the wake of the first issue of the OJAPr. The OJAPr is a new journal in SCIRP's publishing portfolio, and comes as a natural addition to already present journals pertinent to communications and computer sciences, such as the Int'l Journal of Communications, Network and System Sciences (IJCNS), the E-Health Telecommunication Systems and Networks (ETSN), or the Wireless Engineering and Technology (WET) journal.

Joining the growing trend of open-access journals, that has gained popularity in recent years, the OJAPr has also adopted the policy of open-access publishing, enabling the contributing authors to disseminate their works to a substantially larger public in comparison to membership-based journals and at the same time retain the copyright on their manuscript, while interested readers have a free access to published papers.

In today's world of fast-paced technological advances in wireless communication devices and services, one component, amongst many other important components, makes it an inevitable part-an antenna. All who know anything about antennas are familiar with their subtle nature: on one hand, a randomly sized and shaped piece of a conducting wire can eventually act as an antenna working at some frequency band and receive or transmit radio waves successfully enough, while, on the other hand, an exact and thorough understanding of antenna parameters and behavior quickly gets complicated and challenging when trying to mathematically describe its characteristics and successfully design it for a specific goal, or computationally analyze it, manufacture it and assemble precisely and finally verify its desired characteristics by adequate measurements.

Nowadays, we enjoy the commodity of powerful personal computers and advanced computer aided engineering (CAE) software tools to model and predict antenna characteristics by making use of full-wave solvers that are based on some of proven numerical methods, such as the finite-difference time-domain method or the finite element method, and often being empowered by some of popular optimization algorithms, such as the genetic algorithm or the particle swarm optimization algorithm, to enable fine tuning of an antenna design in order to meet specific goals, yet in spite of that, it is still not an easy task to design an antenna that will meet specifications of modern communication devices and services, due to an increased number of goals that are to be met. To be able to transfer data using various wireless technologies, for example GSM, WCDMA, UMTS, WLAN, Bluetooth, NFC, WiMAX, or LTE, modern wireless devices (e.g. smartphones, tablets, or laptops) must be equipped with antennas that can efficiently operate at multiple frequency bands and that goal requires innovative antenna designs that have not been part of classical antenna textbooks and practice. Thus, to meet the required frequency ranges and also fit an antenna into a fairly small space that is left for it in today's feature-packed devices, present-day antennas are often designed using some combination slots or slits, electronic switches such as PIN diodes or MEMS (microelectromechanical switches), vias, and meandering microstrip lines lying on top of the substrate, thus producing the so-called frequency-, polarization-, or radiation pattern-agile antennas, which can only be accomplished by an extensive use of CAE tools in order to predict their performance in a sufficiently accurate and time-efficient fashion.

On the base station side of an RF link, present challenges are related to implementing techniques for more efficient usage of the available spectrum and a need for an increased channel capacity. As the number of wirelessly connected gadgets undergoes a strong growth every year, there is a clear need to secure more bandwidth to accommodate for the growing data amounts. Very soon in the future will the wireless networks have to pass the data amounts that are manifold larger from what we transfer today. Wireless providers are in need for more bandwidth and that will call for a new shift towards higher carrier frequencies. There are multiple as- 
pects involved in research and development (R\&D) efforts tackling those issues and the ultimate success is anticipated to be accomplished by no single means, but rather by judiciously combining multiple techniques as discussed next.

One aspect of improving the quality of service and increasing the capacity lies in implementation of smart(er) antenna arrays on the base station towers. Although the principle of electronic beam scanning has been known for years in radar systems and there has been a good deal of research tackling some sort of smart antennas, it seems that they still have not been adopted in base stations towers to a proportional degree and one reason for that certainly lies in an increased cost that wireless providers would have faced to implement them in their towers due to an increased complexity of such solutions. Smart antennas has been a term denoting antenna arrays that can electronically adjust their radiation pattern to follow select mobile station(s) or/and reduce the level of interference from undesired sources. Depending on the specific goals that were set in the pertinent works, there is a variety of alternative terms that are close to the term "smart antennas". Most commonly they are referred to as the beam-forming arrays, adaptive antennas (or adaptive arrays), up to a more recent terms of self-adaptive arrays and self-recoverable antennas.

Additionally, channel capacity can be increased by making use of polarization agility or diversity by having a set of orthogonally polarized antennas, or using electronic switches to switch between the orthogonal polarizations. While increasing the channel capacity has so far been addressed to the base station side, it can be expected in the future that part of that process will be assigned to mobile handset antennas as well by making them smarter and reconfigurable and that R\&D has been underway for some time now, too.

Yet another known way to increase the network capacity was to reduce the cell size of a wireless network, while at the same time helping mobile handsets work with a lower output power, thus reducing the specific absorption ratio (SAR) that a broad public is getting more sensitive to in the context of possible health effects of radio waves.

Since the quantity of data being transferred over wireless networks is growing fast every year, due to a fastgrowing number of wireless devices and users requesting always-on connection to their emails, websites, social networks, for playing or sharing videos on-the-go, current 4G networks are, or will soon be, about their maximum capacity and will have to be replaced with faster networks containing more bandwidth. Due to that, it is expected that 5G networks will have to be defined and implemented by about 2020. Research efforts in that sense are already underway (see, e.g., Samsung R\&D, the METIS project in Europe, or the WiGig alliance). The major directions of that R\&D will likely include a further reduction of the cell size, thereby forming the so-called pico cells or femtocells and by moving the carrier frequency to a millimeter-wave range, yet simultaneosly retaining the request for having multi-band antennas that will have to cover more than eight frequency bands in order to support all wireless standards. It is possible than novel modulation schemes will also be investigated in order to support the overall change towards 5G standards. As devices working in the millimeter-wave range will exhibit a shorter range to the base station than current mobile handsets, as higher frequencies are prone to higher attenuation, additional base stations will have to be "inserted" into the existing 4G networks. Because of that, it can be expected that additional research will take place searching for more accurate models in describing a communication channel under such condition, where one small niche of that will be about the wireless networks of future airplanes, that are anticipated to replace a sizeable portion of current wiring with wireless networks, to make the airplanes lighter and offer personal communications and entertainment services available to all passengers without compromising safety of plane navigation.

Another important area of $R \& D$ nowadays includes terahertz frequencies. Applications related to them typically involve various noninvasive safety systems like those for airport passenger screening, or detection of explosives, early detection of cracks in the solid materials etc. Terahertz frequencies and applications have gained interest in recent years for their ability to penetrate into materials and trigger their distinct spectral signature that can be used to detect certain substances or warn on possible cracks in the material and more $R \& D$ can be anticipated in that direction to take a full advantage of their promising traits.

Taking into account the whole package of having to achieve a further progress in reconfigurable multi-band antennas that will be even smaller than the present-day antennas, due to a shift in operating frequencies towards $60 \mathrm{GHz}$ and higher, along with the anticipated changes in the network infrastructure, and new conditions in the future communication channels, the upcoming years aiming for 2020 (or as close to it as possible) offer plenty of R\&D excitement in the wireless technology arena and the goal to attain wireless data rates in the order of giga bits per second, which will be 10 to 100 times more than we can afford today.

Having all the aforementioned in mind, establishing a journal specializing in antennas and propagation was a logical next step for SCIRP. The OJAPr invites researchers to submit their original research work for possible publication, whose focus matches the general aims of the journal—discussing ideas and research results pertinent 
to analysis, design, development, optimization, measurement techniques, and applications of antennas and propagations models. The scope of the journal falls within a wide range of categories including, but not limited to, Active and Adaptive Antennas, Antennas Analysis and Design, Beam Control and Steering, Channel Modeling, Intelligent Antennas, Millimeter-Wave Techniques, or Radio Wave Propagation. For a more detailed list of topics please visit the OJAPr website. All submitted manuscripts will undergo a rigorous peer-review process being conducted by the OJAPr Editorial Board, which is staffed by experienced international researchers. The OJAPr office is thankful to its first Editorial Board members who readily offered their service to the journal and will be helping the manuscript submission and review process, namely: Prof. Ahmed M. Attiya as Editor-in-Chief, and Prof. Federico Alimenti, Dr. Haroldo T. Hattori, Prof. M. Ali Hooshyar, Dr. Miroslav Joler, Dr. Chi-Wah Kok, Prof. Igor V. Minin, Dr. Vaclav Papez, Dr.
Càndid Reig, Dr. Zaharias D. Zaharis, and Dr. Domenico Zito, as Editorial Board members, listed in an alphabetical order.

Again, on behalf of the Editorial Board members, myself, and all of the OJAPr staff members who will be serving the publication process, it is my pleasure to invite researchers to prepare and submit to SCIRP's OJAPr manuscripts describing their original research results for possible publication. Short reports and book reviews are also welcome, subject to OJAPr aims, scope, particular topics, and manuscript preparation guidelines that will be maintained on the OJAPr web website.
Sincerely, Miroslav Joler, Ph.D. OJAPr Editorial Board Member 